\title{
BASES FARMACOLÓGICAS DA UTILIZAÇÃO DOS BLOQUEADORES ADRENÉRGICOS BETA NA TERAPÊUTICA DA HIPERTENSÃO ARTERIAL
}

\author{
Virgílio Durão
}

Instituto de Farmacologia. Faculdade de Medicina de Lisboa. Lisboa.

A utilização dos fármacos bloqueadores dos receptores adrenérgicos beta no tratamento da HTA, generalizou-se após a publicação das primeiras observações clínicas por Prichard em 1964, sobre o efeito anti-hipertensor do pronetalol. Trabalhos subsequentes demonstraram que a maioria dos bloqueadores $\beta$, como o propranolol (Prichard e Gillam 1964), o pindolol (Thorpe 1972), o oxprenolol (Leishman et al 1970), o alprenolol (Furberg e Michaelson 1969), o sotalol (Prichard e Boakes 1974), etc., possuiam esta actividade anti-hipertensora, existindo hoje uma extensa bibliografia que comprova, não só a sua eficácia terapêutica como também a pequena incidência de efeitos secundários nos doentes hipertensos tratados (Doyle 1974; Simpson 1974; Dollery 1977). Estas características levaram alguns Autores a propor os bloqueadores beta como anti-hipertensores de $1 .^{a}$ linha, mau grado o seu mecanismo de acção não estar completamente esclarecido.

Enquanto que, sob um ponto de vista farmacológico, à luz da teoria dos receptores celulares, o efeito anti-hipertensor dos bloqueadores dos receptores adrenérgicos alfa é facilmente previsível se atendermos à distribuição não uniforme destes receptores no sistema cardio-vascular, o mesmo não acontece em relação aos bloqueadores beta. $\mathrm{Na}$ figura 1 pode ver-se que os receptores alfa predominam no músculo liso das veias e das arteriolas pré-capilares do organismo, com excepção das arteríolas nutritivas dos músculos estriados voluntários. A sua activação por um fármaco agonista, como a nor-adrenalina, origina uma contraç̧ão do músculo liso arteriolar, que por sua vez condiciona um aumento das resistências vasculares periféricas com a resultante subida da pressão arterial. Inversamente, o bloqueio destes receptores alfa por uma droga antagonista, dá origem a uma cadeia de fenómenos inversa: relaxamento da musculatura lisa arteriolar, diminuição dạs resistências vasculares periféricas e descida da pressão arterial. Estas acções explicam o efeito anti-hipertensor dos bloqueadores alfa e justificam teoricamente a sua utilização na terapêutica da hipertensão arterial.

Uma explicação teórica semelhante não é aplicável à actividade anti-hipertensora dos bloqueadores beta. Com efeito, se atendermos à distribuição dos receptores beta e às acçōes resultantes da sua activação, representadas na figura 1 , facilmente se verifica que um agonista, como a isoprenalina, provoca um relaxamento do músculo liso das arteríolas musculares, com diminuição das resistências periféricas e um aumento do débito cardíaco, originando no animal de experiência uma hipotensão moderada. Um efeito oposto, com uma ligeira subida da pressão arterial seria pois previsível quando 
os receptores beta são bloqueados por um fármaco antagonista. $\mathrm{Na}$ realidade a acção final dos bloqueadores beta sobre a pressão arterial é variável com o modelo experimental e a espécie animal utilizados, sendo muito difícil de demonstrar na prática (Clark 1976). Como exc-j̧̧̃es apontam-se o rato normal onde se verifica uma hipertensão, e o rato hipertenso e o coelho consciente, não anestesiado, onde recentemente foi possivel evidenciar um efeito hipotensor (Weber et al 1974; Takeda et al 1975; Durão e Rico 1977).

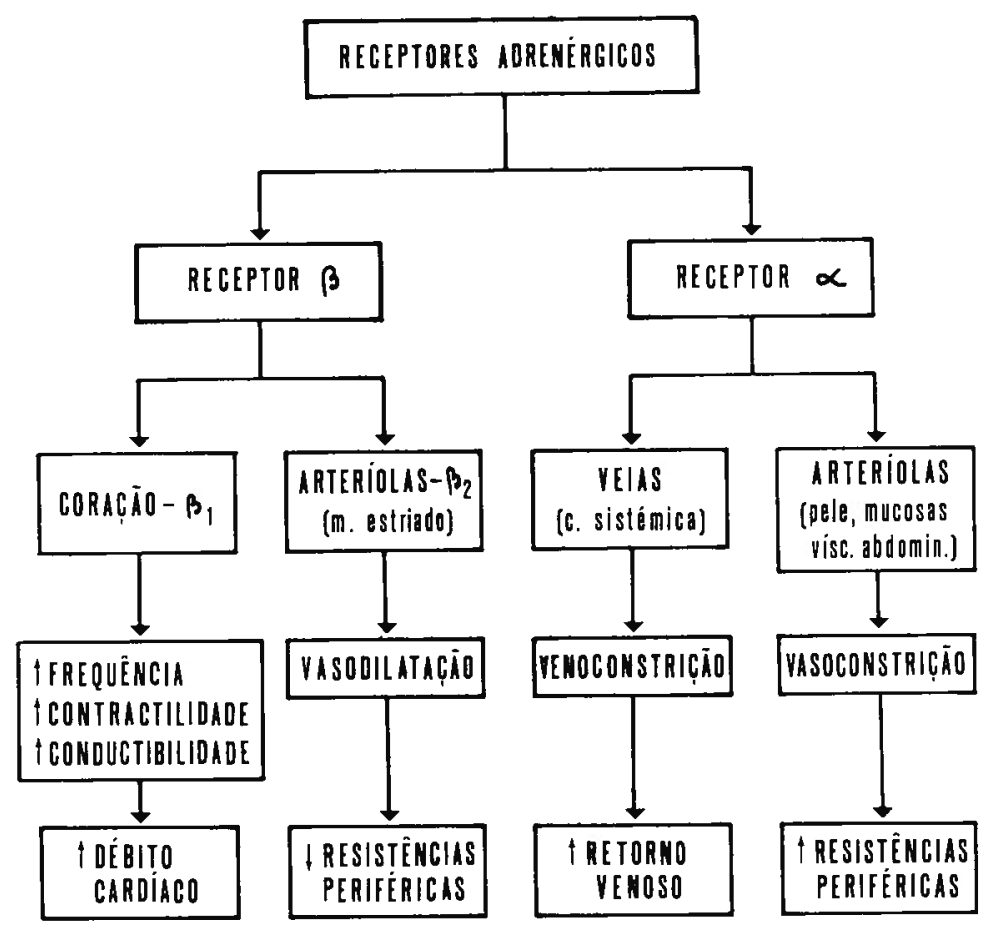

Fig. 1 - Esquema da distribuição dor receptores adrenérgicos no sistenta cardio- vascular $e$ efeitos farmacológicos da sua activaşäo

Estes factos levaram alguns Autores como Farmer e Levy (1968) a afirmar, em meados da década de 60 , que os resultados obtidos em animais bipertensos levam-nos a pensar que o propranolol e outros bloqueadores $\beta$, não devem comportar-se como agentes anti-bipertensores eficazes no bomem.

Actualmente, mais de dez anos decorridos, subsiste o desafio aos farmacologistas: explicar, à luz da teoria dos receptores celulares, e demonstrar experimentalmente, o mecanismo da acção anti-hipertensora dos bloqueadores beta, já exaustivamente comprovada na prática clínica.

A resolução deste problema complica-se ainda mais quando se constata que os numerosos bloqueadores beta actualmente disponíveis, nāo constituem um grupo homogéneo e possuem outras propriedades farmacológicas que os distinguem entre si. Entre estas propriedades contam-se as estabilizadoras da membrana de tipo quinidina, a actividade simpaticomimética intrínseca, a cardioselectividade atribuída a alguns e a lipossolubilidade que condiciona a passagem da barreira hemato-encefálica (Quadro I). 


\section{QUADRO I}

Propriedades farmacológicas de alguns bloqueadores adrenérgicos beta

\begin{tabular}{|c|c|c|c|c|c|}
\hline & $\begin{array}{l}\text { Potência do } \\
\text { bloqueio B }\end{array}$ & $\begin{array}{c}\text { Estabilização } \\
\text { da membrana } \\
\text { (quinidine-like) }\end{array}$ & $\begin{array}{c}\text { Actividade } \\
\text { simpaticomimétic: } \\
\text { intrinseca }\end{array}$ & $\begin{array}{l}\text { Especificidade } \\
\text { para } \\
\text { receptores Bl }\end{array}$ & $\begin{array}{c}\text { Pasagem da } \\
\text { barreira } \\
\text { hemato-encefálica }\end{array}$ \\
\hline Oxprenolol & 2.0 & ++ & ++ & - & ++ \\
\hline Alprenolol & 1.0 & $+t$ & ++ & - & ++ \\
\hline Acebutolol & 3.0 & + & + & + & \\
\hline Propranolol & 1.0 & ++ & - & - & ++ \\
\hline Timolol & & + & - & - & ++ \\
\hline Pindolol & 5.0 & - & + & - & ++ \\
\hline Practolol & 0.5 & - & + & ++ & +- \\
\hline Sotalol & 0.1 & - & - & - & +- \\
\hline Atenolol & 2.5 & - & - & ++ & +- \\
\hline
\end{tabular}

A existência destes diferentes espectros de aç̧ão farmacológica exacerbou a polémica já existente e, aliada às dificuldades teóricas e experimentais já apontadas, deu origem a numerosas teorias que propõem mecanismos de acção variados e díspares para explicar a actividade anti-hipertensora dos bloqueadores beta.

$\mathrm{Na}$ figura 2 estão representados os cinco mecanismos mais frequentemente propostos.

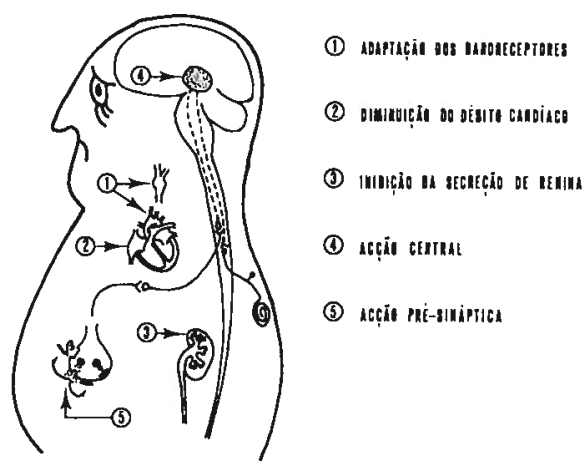

Fig. 22 - Mecanismos mais frequentemente propostos para a aç̧ăo anti-bipertensora dos bloqueadores $\beta$ 
1. Adaptação dos baroreceptores - proposto inicialmente por Prichard e Gillam (1969) atribuía a baixa de pressão arterial a uma diminuição dos reflexos pressores mediados pelo simpático e uma adaptação progressiva dos baroreceptores para níveis de pressão mais baixos. Não foi possível confirmar esta hipótese em estudos experimentais no animal (Kelleher et al 1972) ou no homem (Ulrych 1969), estando hoje praticamente abandonada.

2. Diminuição do débito cardíaco - esta acção, resultante do bloqueio dos receptores $\beta$ cardíacos, não é constante para todos os bloqueadores beta, sendo mais marcada com os que possuem actividade estabilizadora da membrana e deprimem inespecificamente a fibra miocárdica, como o propranolol. O pindolol e outros antagonistas $\beta$, como - oxprenolol e o alprenolol, que possuem actividade simpaticomimética intrínseca, não reduzem significativamente o débito cardíaco, não podendo esta hipótese ser invocada para explicar o seu efeito anti-hipertensor (Giudicelli et al 1969; Barret, 1970; Bergamaschi et al 1971). Por outro lado, mesmo os bloqueadores beta que provocam uma diminuição acentuada do débito, originam simultaneamente, por bloqueio dos receptores $\beta$ vasculares e por mecanismo reflexo, um aumento das resistências vasculares periféricas (Fig. 1), que anulam o efeito hipotensor resultante da baixa do débito cardíaco (Mckenna et al 1966; Barret 1970).

Também nos estudos com modelos animais de hipertensão, existe sempre uma dissociação entre o efeito sobre a pressão arterial e as alterações da frequência e débito cardíacos, provocadas pela administração aguda, em injecção única, ou prolongada dos bloqueadores beta (Lee e Simpson 1973; Dusting e Rand 1974). Nós próprios confirmámos, no coelho consciente, que à diminuição inicial da frequência cardíaca provocada pelo propranolol, se associa uma ligeira subida da PA, verificando-se o efeito hipotensor apenas mais tardiamente, quando a frequência retoma valores próximos do normal. No mesmo modelo, o pindolol, origina uma diminuiçāo da PA, sem alteraçōes significativas da frequência (Durão e Rico 1977).

Parece-nos lícito concluir que a redução do débito cardíaco, se bem que possa ser um factor complementar no efeito hipotensor de alguns bloqueadores $\beta$, não é uma explicação plausível para a actividade anti-hipertensora destes fármacos (Clark 1976).

3. Inibição da secreção de renina - Uma interferência dos bloqueadores $\beta$ no sistema renina-angiotensina-aldosterona tem sido insistentemente proposta por alguns $\mathrm{Au}$ tores, que se apoiaram sobretudo em resultados obtidos em estudos de farmacologia clínica no homem (Buhler et al 1972; Michelakis et al 1972). A base teórica desta hipótese assenta na acção estimulante do sistema nervoso simpático sobre a secreção de renina, sendo este ef eito mediado por receptores $\beta$ (Weber et al $1974 \mathrm{a}$ ). Assim, o bloqueio destes receptores origina uma diminuição da libertação de renina, com redução da angiotensina II circulante e consequente baixa da PA.

O grupo de Laragh, não só sugere esta actividade anti-renina como mecanismo da acção anti-hipertensora dos bloqueadores $\beta$, como também propõe a medição da actividade renina plasmática como meio de seleccionar os doentes e prever a sua resposta a esta terapêutica (Laragh 1973). Esta teoria não resistiu no entanto à comprovação experimental a que a submeteram outros Autores, e está actualmente reduzida às suas verdadeiras dimensões, aplicando-se apenas a alguns doentes em situações clínicas bem definidas.

Com efeito, experiências laboratoriais com modelos animais, demonstram que os bloqueadores $\beta$ apenas inibem a secreção de renina provocada por estimulação adrenérgica, não alterando a libertação provocada por outros estímulos que actuariam por mecanismos diferentes, como redução da pressão de perfusão renal, hemorragia, etc. (Imbs et al 1974). 
Também Weber e colaboradores (1974 b) demonstraram no coelho que é possível obter um efeito hipotensor sem alteração da actividade renina plasmática, ou mesmo obter uma diminuição da pressão arterial com aumento simultâneo da secreção de renina, como verificaram com o pindolol.

Por outro lado, estudos clínicos muito numerosos, se bem que fornecendo por vezes dados contraditórios, provavelmente resultantes de metodologias diferentes, mostram também que não existe uma correlação entre a inibição da secreção de renina e o efeito anti-hipertensor (Stokes et al 1974; Chalmers et al 1976; Woods et al 1976). Apenas tem sido possível, em alguns casos, demonstrar uma correlação entre os niveis iniciais de renina e o efeito terapêutico dos bloqueadores $\beta$ (Buhler et al 1973). Tivemos ocasião de comprovar estes factos num ensaio clínico de colaboração com Martins Prata e J. Barbas, em que o bloqueador $\beta$ utilizado, o pindolol, originou um efeito anti-hipertensor eficaz em todos os doentes, sem alterações significativas da actividade renina plasmática (Prata et al 1977).

Consideramos assim que não é possível explicar a eficácia terapêtica destes compostos, na maioria dos doentes hipertensos, por um mecanismo anti-renina.

4. Mecanismo de acção central - esta hipótese apoia-se sobre a observação experimental duma queda tensional acompanhada de bradicárdia após a injecção de bloqueadores $\beta$ nos ventrículos cerebrais (Dollery et al 1973; Carter et al 1974).

A importância dos mecanismos adrenérgicos centrais na regulação da pressão arterial está hoje bem estabelecida, tendo sido demonstrada uma acção inibitória mediada por receptores alfa (Van Zwieten 1973). O papel dos receptores $\beta$ neste mecanismo de regulação central é mais nebuloso, verificando-se respostas tensionais variáveis após a injecção intra-ventricular de isoprenalina (Day e Roach 1974). A hipotensão e a bradicárdia resultantes da administração central dos antagonistas $\beta$ é mais constante, tendo sido comprovada para a maioria destes compostos actualmente disponíveis, e indiscutivelmente associada ao bloqueio dos receptores $\beta$ centrais. Também recentemente foi demonstrado que este efeito central é simultâneo com uma diminuição da actividade simpática pré-ganglionar que, segundo alguns Autores, mediaria o efeito hipotensor e a bradicárdia observados (Lewis e Heusler 1975). No entanto, esta redução do tónus simpático periférico não se verifica após a administração intravenosa do propranolol e do pindolol, levando alguns Autores a duvidar da importância dos efeitos centrais no mecanismo de acção dos bloqueadores $\beta$ (Clark 1976).

Uma tal acção central não pode aliás ser invocada para alguns destes fármacos, como o practolol e o sotalol que, embora possuindo actividade anti-hipertensora, atravessam com dificuldade a barreira hemato-encefálica devido à sua reduzida lipossolubilidade (Garvey e Ram 1975).

5. Inibição da transmissão adrenérgica por acção pré-sináptica - esta hipótese foi inicialmente proposta para alguns bloqueadores $\beta$ que possuem actividade anestésica local e que provocam um efeito estabilizador da membrana. Este efeito pode produzir uma inibição da recaptação de nor-adrenalina pelas terminações nervosas pré-sinápticas, assim como uma inibição da sua libertação ao nível da sinapse. Como consequência, resultaria uma depleção dos depósitos do mediador adrenérgico e uma redução da quantidade libertada por impulso, que acarretaria uma diminuição da actividade simpática.

Recentemente, os trabalhos de Langer e colaboradores (Adler-Graschinsky e Langer 1975; Langer 1977), trouxeram uma explicação adicional para este efeito inibidor da transmissão simpática. Segundo este Autor, existem receptores pré-sinápticos que têm como função modular, por um mecanismo de retroacção, a libertação da nor-adrenalina; um receptor alfa que inibe a libertação e um receptor beta que, quando activado, aumenta 
a quantidade de mediador libertada. O bloqueio deste receptor $\beta$ teria o efeito oposto e originaria uma diminuição da nor-adrenalina que se liberta por impulso.

Esta teoria está já hoje largamente comprovada experimentalmente e, se os receptores pré-sinápticos existirem também nos neurónios simpáticos que inervam as arteríolas pré-capilares, como parece provável, este mecanismo de inibição da transmissão adrenérgica pode reduzir o tono arteriolar e contribuir para o efeito anti-hipertensor dos bloqueadores $\beta$. Aliás, nos últimos anos, foram publicados alguns trabalhos de farmacologia clínica que sugerem que alguns destes mecanismos podem concorrer para a eficácia terapêutica dos bloqueadores $\beta$ no homem. Bbrecht e colaboradores $(1975,1976)$ demonstraram, em doentes hipertensos, que o pindolol reduz, após um período de tratamento prolongado, os níveis de nor-adrenalina plasmática, que se encontravam inicialmente elevados. Esler (1977) comprovou que os doentes com catecolaminas altas tinham uma resposta hemodinâmica particularmente favorável ao propranolol.

Se aceitarmos, com algumas reservas, que a quantidade de nor-adrenalina circulante pode ser tomada como um índice da actividade do sistema adrenérgico, estes dados confirmam, na prática clínica, a redução da actividade simpática periférica, postulada nas hipóteses experimentais que propõem um mecanismo de acção central e uma acçäo inibidora da transmissão adrenérgica pré-sináptica. Também, como já vimos atrás, o efeito inibidor da secreção de renina pode contribuir para o efeito hipotensor dos bloqueadores $\beta$ em alguns doentes, sobretudo nos que têm niveis muito elevados de angiotensina.

A nosso ver, qualquer destes mecanismos considerado isoladamente não consegue explicar a actividade terapêutica dos bloqueadores $\beta \mathrm{em}$ todas as situações e em todos os doentes. Um dos erros dos Autores que os propuseram foi tentar explicar o efeito anti-hipertensor destes compostos, que é necessariamente complexo, por um mecanismo único. Pensamos como Clark (1976) que os mecanismos envolvidos devem ser múltiplos e actuar simultâneamente.

Por outro lado, os investigadores têm-se preocupado apenas com a interaç̧ão dos bloqueadores $\beta$ com os sistemas hipertensores fisiológicos que regulam a pressão arterial normal. Todas as hipóteses que analisámos atrás envolvem os mecanismos pressores, existindo uma bibliografia extensa e exaustiva sobre a acção do bloqueio beta nos sistemas adrenérgico e renina-angiotensina-aldosterona. Surpreendentemente pouca atenção tem sido prestada aos mecanismos hipotensores, nomeadamente as prostaglandinas endógenas, e à sua possível função na actividade anti-hipertensora dos bloqueadores $\beta$.

A pressão arterial normal resulta do equilíbrio entre mecanismos hipertensores e hipotensores, que se antagonizam mutuamente (fig. 3). A importância dos sistemas hipotensores humorais é conhecida desde as já clássicas experiências de Goldblatt (1934). Este Autor demonstrou que é mais fácil obter uma hipertensão reno-vascular experimental por constrição unilateral de uma artéria renal quando se remove o rim são, contra-lateral, do que quando se deixa este in situ. A substância produzida pelo rim são e que se opõe, por um efeito hipotensor, ao desenvolvimento da hipertensão experimental foi denominada medulina. Posteriormente, Lee $(1962 ; 1963)$ verificou que o extracto de medula renal de coelho se comportava como um hipotensor potente em vários modelos laboratoriais, utilizando animais normais e hipertensos. Este Autor demonstrou também que a medulina tinha uma actividade anti-hipertensora muito acentuada no homem, identificando mais tarde como prostaglandinas A e E as substâncias activas dos extractos medulares responsáveis pelos efeitos hipotensores (Lee 1967; Lee et al 1965, 1971).

As prostaglandinas são substâncias de estrutura complexa, derivadas de ácidos gordos essenciais, com uma distribuição quase ubiquitária em todo o organismo e com 
um espectro farmacológico larguíssimo, que abrange praticamente todas as funçōes biológicas (Douglas 1975). Dividem-se em vários grupos - E, F, A, B, C e D - con-

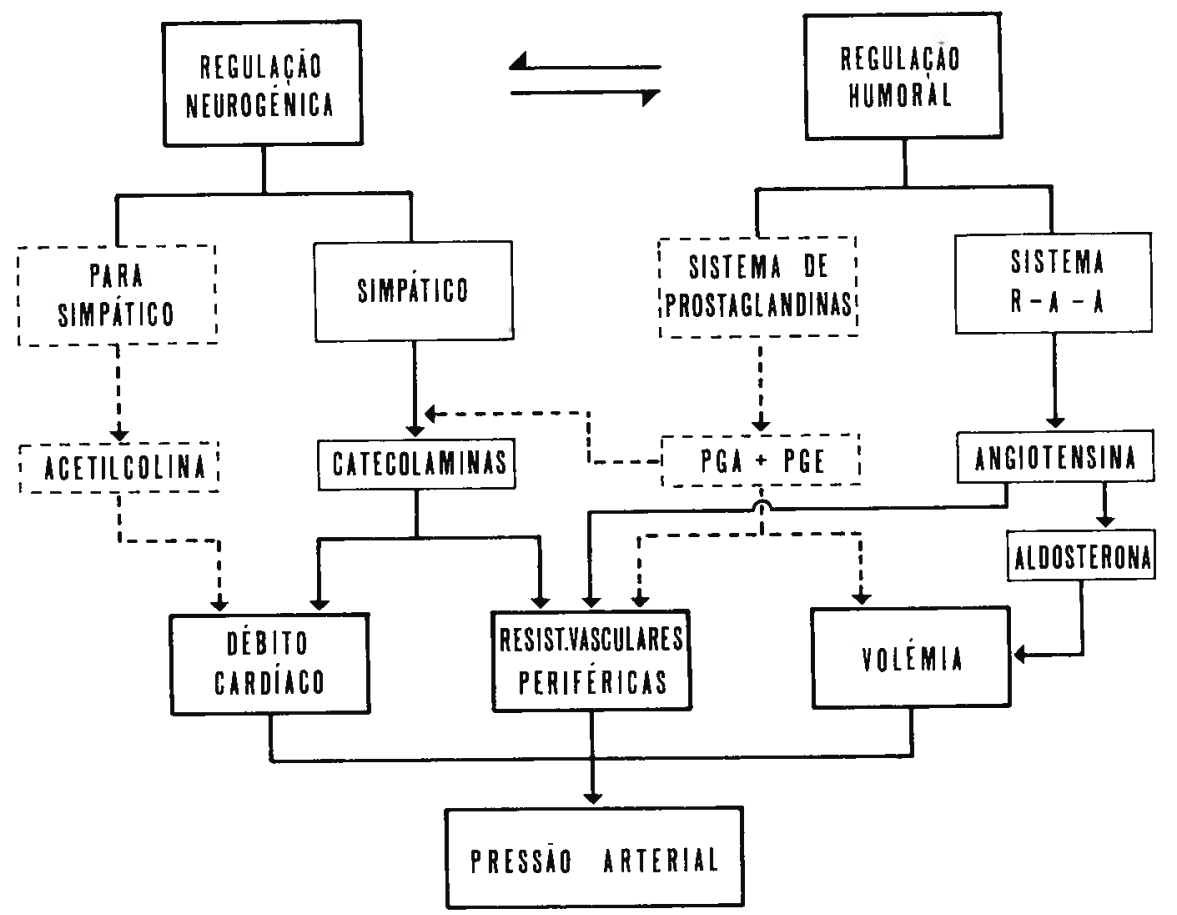

Fig. 3-Esquema simplificado dos mecanismos de regulaşāo da pressão arterial. Sistemas bipertensores representados a traço continuo e sistemas bipotensores a tracejado

forme a estrutura do anel ciclopentano, subdividindo-se depois os grupos principais em sub-grupos conforme o ácido gordo percursor, o que é indicado pelo índice numérico 1, 2 ou 3. Por exemplo, as prostaglandinas $E_{2}$ e $F_{2} \alpha\left(P_{2} E_{2}, P G F_{2} \alpha\right)$ derivam do ácido araquidónico. As prostaglandinas dos grupos $\mathrm{E}$ e $\mathrm{F}$, que são também denominadas prostaglandinas primárias, são as mais abundantes no organismo e dão origem às prostaglandinas dos outros grupos (fig. 4).

Em relação ao sistema cardio-vascular, as PGE e PGA comportam-se como hipotensores poderosos que provocam uma vasodilatação das arteríolas pré-capilares e uma redução marcada das resistências vasculares periféricas. Em trabalhos recentes, Lee (1974) salientou a sua importância na etiopatogenia da hipertensão arterial. De acordo com este Autor, alguns quadros de hipertensão nos animais e no homem resultam não só de uma actividade excessiva dos sistemas pressores, mas também de uma deficiência dos mecanismos hipotensores, nomeadamente as PGA e PGE renais, que pode permitir 
aos mecanismos hipertensores actuar sem oposição e originar uma situação hipertensiva (Lee et al 1976).

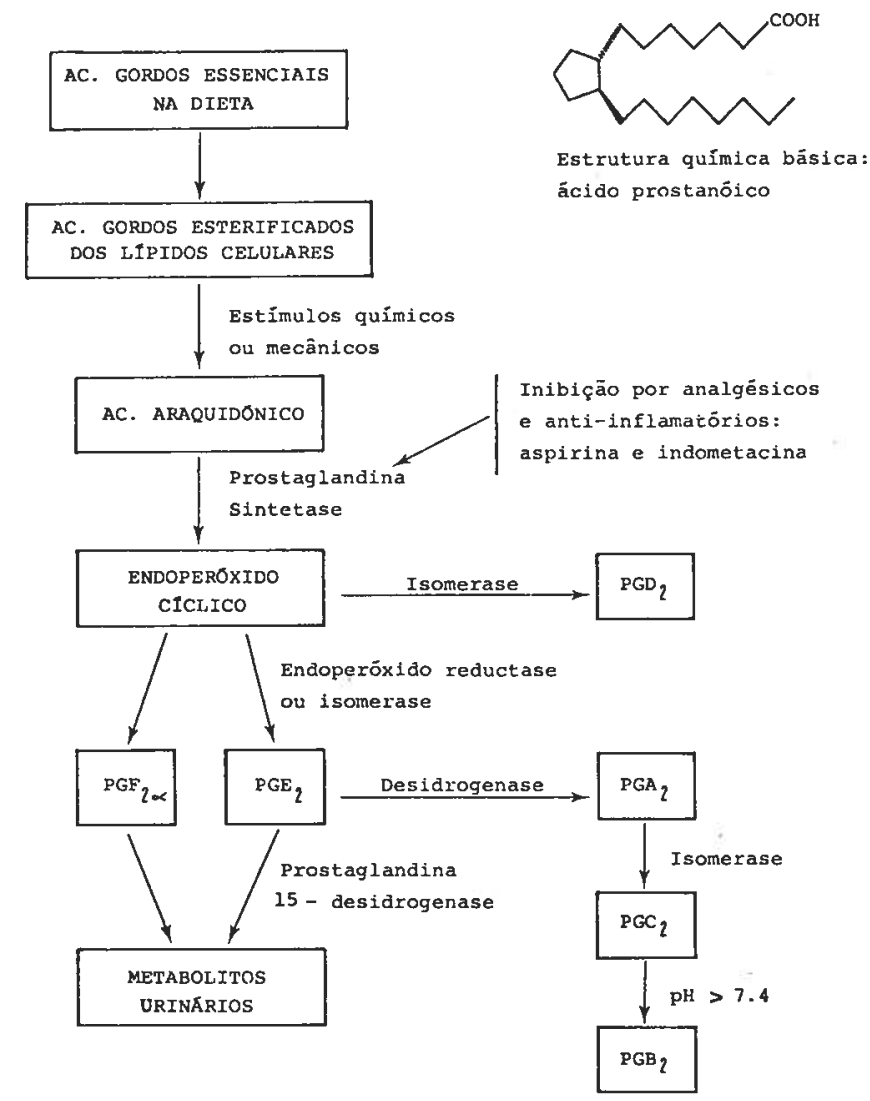

Fig. 4-Esquema do metabolismo das prostaglandinas

Fundamentados nestas observações $e$ atendendo ao facto de a interaç̧ão dos bloqueadores $\beta$ com os sistemas hipertensores não clarificar decisivamente o seu mecanismo de acção, delineámos um protocolo experimental com o intuito de verificar a possível influência dos mecanismos hipotensores, sobretudo as prostaglandinas endógenas, na actividade anti-hipertensora daqueles compostos.

Utilizámos como modelo laboratorial o coelho consciente, com monitorização contínua da pressão arterial e frequência cardíaca (Durão e Rico 1977). Nestas condições 
experimentais a administração i. v. de pindolol e de propranolol provocou uma diminuição altamente significativa da pressão arterial média (Fig. 5). Salientamos que este

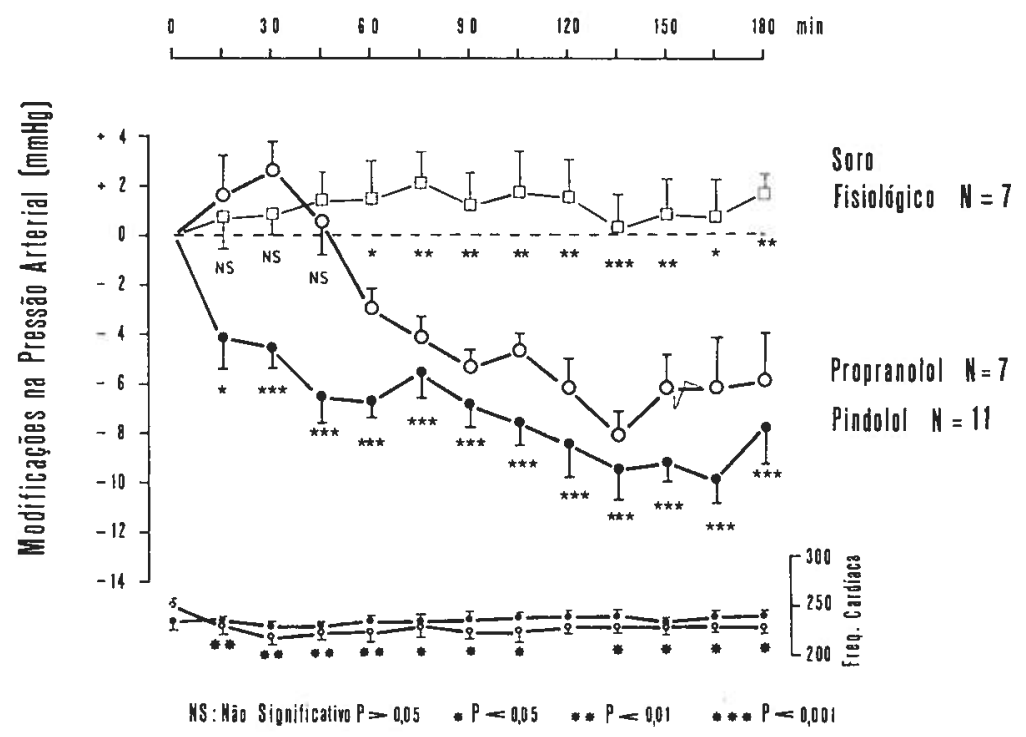

Fig. 5-Efeito da administraçäo IV de propranolol e pindolol na pressão arterial média e frequência cardíaca de coelhos conscientes normais. Obtiveram-se efeitos bipotensores altamente significativos (test-t de student) com ambos os fármacos

efeito hipotensor foi obtido com o pindolol sem alterações significativas da frequência cardíaca, verificando-se com o propranolol uma bradicárdia inicial coincidente com uma ligeira elevação da pressão arterial. Como já afirmámos antes, estes resultados confirmam que a bradicárdia e a diminuição do débito cardíaco não devem contribuir significativamente para a aç̧ão hipotensora observada. Noutro grupo de experiências, foram injectadas as mesmas doses dos bloqueadores $\beta$ em animais previamente tratados com dois fármacos inibidores da síntese das prostaglandinas, a indometacina e o ácido acetilsalicílico.

Os resultados obtidos (fig. 6) mostram que a inibição das prostaglandinas endógenas anulou o efeito hipotensor dos dois bloqueadores beta ensaiados, levando-nos a concluir que aquelas substâncias estão de algum modo relacionadas com a diminuição da pressão arterial observada na fig. 5. 


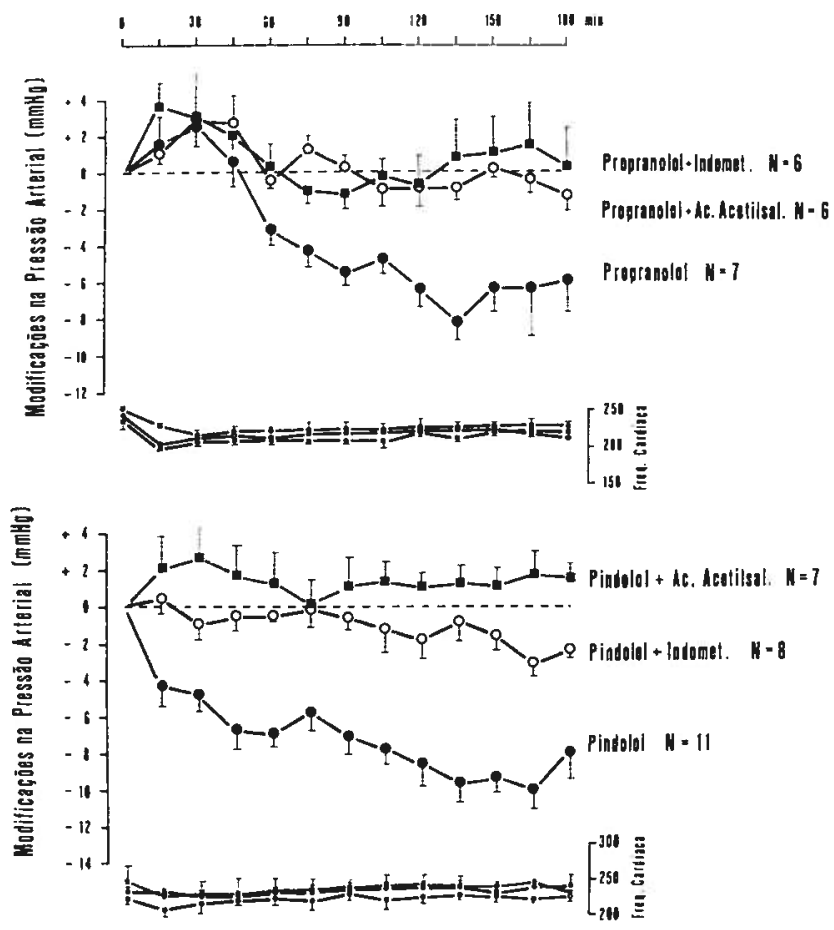

Fig. $6-$ Abolição do efeito bipotensor do pindolol e do propra. nolol no coelbo consciente pela inibicäo da sintese das prostaglan. dinar pela indometarina e ácido acetilsalicilics

Esta hipótese experimental foi depois investigada num estudo de farmacologia clínica delineado com o fim de determinar se a actividade anti-hipertensora dos mesmos bloqueadores $\beta$ em doentes hipertensos era analogamente modificada quando se inibia a síntese das prostaglandinas pela indometacina (Durão et al 1977).

A pressão arterial de todos os doentes incluídos no estudo teve um comportamento semelhante, independentemente do bloqueador $\beta$ utilizado, verificando-se que a inibição das prostaglandinas endógenas aboliu significativamente o efeito anti-hipertensor (fig. 7). A pressão arterial só retoma os valores normais iniciais quando a indometacina é retirada do regimen terapêutico. Estes dados clínicos confirmam os resultados obtidos no animal consciente e permitem-nos afirmar que, também nos doentes hipertensos, as prostaglandinas endógenas estão de alguma maneira implicadas na eficácia terapêutica dos bloqueadores beta.

Está hoje comprovado que estes fármacos reduzem a pressão arterial através de uma diminuição gradual das resistências vasculares periféricas, apesar de o mecanismo envolvido nestes efeito vasodilatador ser obscuro (Clark 1976; Ablad et al 1976; Amery et al 1976). Pensamos que as prostaglandinas endógenas, particularmente as PGA e PGE, que como jả vimos são hipotensores potentes que dilatam as arteríolas e reduzem as 
resistências vasculares, podem ser um dos mediadores do efeito vasodilator e consequente diminuição das resistências vasculares periféricas provocada pelos bloqueadores $\beta$. No ensaio clínico já citado atrás, o efeito da inibição da síntese das PGS foi mais acentuado na pressão arterial diastólica, que se manteve praticamente inalterada durante o tratamento combinado com bloqueadores $\beta$ e indometacina (fig. 7). Como a pressão diastólica revela com maior acuidade as alterações das resistências vasculares periféricas (Guédon 1974), os resultados clínicos apresentados estão de acordo com a hipótese exposta.

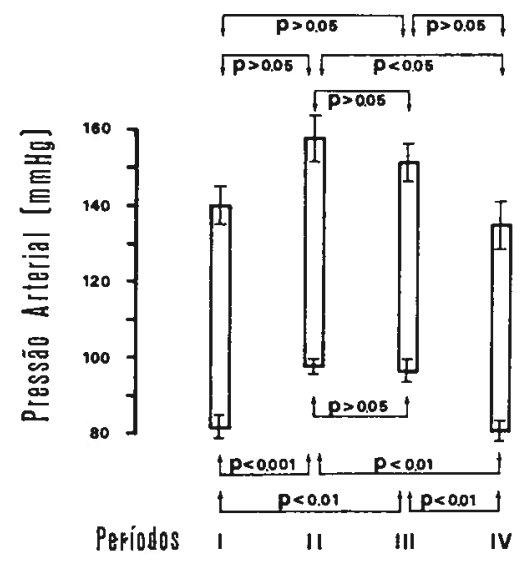

Fig. 7 -Varaçós da pressäo arterial ststolica $e$ diastólica (médial $=$ erro padrüo) de 7 doentes bipertensor durante 4 periodos de tratamento: periodo $l$. bloqueador $\beta$ isolado; $p e$ riodo II, placebo: periodo III. bloqueador $\beta$ $e$ indometacina: período IV. bloqueador $\beta$ isolado. Estudo cistatistico com o test-t de Siudent

Como conclusāo, propornos que o efeito anti-hipertensor dos bloqueadores $\beta$ resulta não só de uma actividade antagonista em relação aos sistemas pressores (como bloqueio da secreção de renina pelo rim ou redução dos impulsos simpáticos para o coração e vasos através de um mecanismo central e de uma inibição da transmissão adrenérgica pré-sináptica) mas também de uma acção sinérgica com os sistemas hipotensores fisiológicos.

E se nos é lícito especular um pouco, pensamos que as caracteristicas da actividade anti-hipertensora dos bloqueadores beta derivam desta multiplicidade de pontos de actuação, envolvendo vários mecanismos. Outros anti-hipertensores, principalmente os que interferem exclusivamente com o simpático, para serem eficazes, provocam necessariamente uma inibição muito marcada deste sistema de regulação da pressão arterial, que deixa assim de contribuir para a adaptação do organismo a situaçōes várias, como o ortostatismo, ou para a execução de outras funções, como função sexual no homem, com todo o cortejo de efeitos secundários que daí advêm. A associação de anti-hipertensores com mecanismos de acção diferentes, prática corrente no tratamento da hipertensão, pretende exactamente obviar a estes inconvenientes (Dollery 1977). 
Os bloqueadores beta, actuando por um lado sinergicamente com os mecanismos hipotensores, e por outro antagonizando dois dos principais mecanismos pressores, o sistema renina-angiotensina e o sistema nervoso simpático, não necessitam provocar uma inibição drástica destes mecanismos, resultando o seu efeito final sobre a pressão arterial do somatório de acçōes parciais moderadas, que permitem àqueles sistemas continuar a assegurar todas as outras funçōes biológicas em que estão envolvidos.

Esta hipótese explica a acção anti-hipertensora moderada, gradualmente progressiva e sobretudo com poucos efeitos secundários, que concorreu para a crescente popularidade dos bloqueadores $\beta$ e para o lugar de destaque que actualmente ocupam no arsenal terapêutico anti-hipertensor.

\section{Agradecimentos}

Agradecemos ao Prof. J. M. Toscano Rico os valiosos comentários críticos que teve a gentileza de fazer ao trabalho e manifestamos o nosso reconhecimento às Sras. D. Isabel Jorge e D. Maria José Lima pelo auxílio prestado na preparação do manuscrito e na execução das figuras.

\section{SUMMARY}

It is now well established that beta-adrenoceptor blockers are effective and safe antihypertensive agents. Despite an extensive use in the therapy of arterial hypertension, often as first-choice treatment, the mechanism by which these drugs lowered blood pressure is still controversial. This is largely due to the difficulties encountered in demonstrating the blood pressure lowering effect in animal experiments and to the fact that blockade of peripheral vascular receptors is paradoxical with the antihypertensive activity. Five different mechanisms have been proposed - resetting of baroreceptors, reduction in cardiac output, central inhibition of sympathetic nerve activity, peripheral reduction of sympathetic transmitter release through a presynaptic action, inhibition of renin release - but none has been confirmed beyond doubt. Much has been published about the interaction of beta-blockers with the pressor mechanisms that control normal blood-pressure, but surprisingly, little attention has been paid to the hypotensive mechanisms - namely the endogenous prostaglandins - and their possible role in the antihypertensive activity of these agents. We have demonstrated in conscious animals that the blood pressure lowering effect of pindolol and propranolol was abolished by the inhibition of prostaglandin synthesis.

In a clinical trial with hypertensive patients we have observed that the antihypertensive activity of these two $\beta$-blockers was also significantly supressed when the prostaglandin synthesis was inhibited by indomethacin.

Our results suggest that the antihypertensive effect of $\beta$-adrenoceptor blocking agents might not be solely the result of an antagonistic activity against pressor systems (such as reduced sympathetic discharge to blood vessels and heart via a central action, reduced release of transmitter from post-synaptic adrenergic neurons, or blockade of renin release from the kidney) but might also result from a synergistic activity with the hypotensive mechanisms. 


\section{BIBLIOGRAFIA}

ABLAD B, LJUNG B, SANNERSTEDT R: Haemodynamic effects of beta-adrenoreceptors blockers in hypertension. Drugs 11: supl 1: 127, 1976.

ADLER-GRASCHINSKY E, LANGER SZ: The possible role of beta-adrenoceptor in the regulation of noradrenaline release by nerve stimulation through a positive feed-back mechanism. Brit I Pharmacol 53: 43, 1975.

AMERY A, BILLIET L, BOEL A, FAGART R, REYBROUCK T, WILLEMS J: Mechanism of hypotensive effect during beta-adrenergic blockade in hypertensive patients, Amer Heart $J$ 91: 634, 1976.

BARRET AM: A survey of the pharmacologic properties of adrenergic beta-receptor antagonists. Int J Clin Pbarmacol 3: Supl 3: 2, 1970.

BERGAMASCHI M, SHANKS RG, CARAVAGGI AM, MANDELLI V: A comparison of the cardiovascular actions of four adrenergic beta-receptor blocking drugs in resting, conscious dogs. Am Heart $\int$ 82: 338, 1971.

BRECHT HM, BANTHIEN F, SCHOEPPE W: Decrease in plasma noradrenaline levels following long-term treatment with prindolol in patients with essential hypertension. Klin Wschr 54 : $1095,1976$.

BRECHT HM, VLACHOYANNIS J, MUSIL HA, ERNST W, WEISMULLER G, SCHOEPPE W: The change in plasma catecholamine concentration and the cyclic AMP excretion after beta-blockade in essential hypertension Verb dtsch Ges inn Med 81: 1035, 1975.

BÜHLER FR, LARAGH JH, BAER L, VAUGHAN ED, BRUNNER HR: Propranolol inhibition of renin secretion - a specific approach to diagnosis and treatment of renin-dependent hypertensive diseases. New Engl I Med 287: 1209, 1972.

BÜHLER FR, LARAGH JH, VAUGHAN ED, BRUNNER HR, GAVRAS H, BAER L: Antihypertensive action of propranolol. Specific anti-renin responses in high and normal renin forms of essential, renal, renovascular, and malignant hypertension. Amer I Cardiol 32: 511 , 1973.

CARTER JK, MITCHELL HW, PYSER RH: Comparison of some haemodynamic changes between central and intravenous administration of (士) propranolol in anaesthetised dogs. Brit I Pbamacol 51: 146 P, 1974

CHALMERS J, HORVATH J, TILLER D, BUNE A: Effects of timolol and hydrochlorothiazide on blood-pressure and plasma renin activity - double-blind factorial trial. Lanct 2: 328, 1976.

CLARK BJ: Pharmacology of beta-adrenoceptor blocking agents, In: $\beta$-Adrenoceptor Blocking Agents, (North Holland, Amsterdam) eds. $P R$ Saxena e $R$ P Forsyt p. 45, 1976.

DAY MD, ROACH AG: Central alpha - and beta-adrenoceptors modifying arterial blood pressure and heart rate in conscious cats. $B r J$ Pharmacol 51: 325, 1974.

DOLLERY CT: Pharmacological basis for combination therapy of hypertension. Ann Rev' Pharmacol Toxicol 17: 311, 1977.

DOLLERY CT, LEWIS PJ, MYERS MG, REID JL: Central hypotensive effect of propranolol in the rabbit. Brit I Pbarmacol 48: $343 \mathrm{P}, 1973$.

DOUGLAS WW: Polypeptides - angiotensin, plasma kinins, and other vasoactive agents; prostaglandins, In: The Pharmacological Basis of Therapeutics, New York Macmillan Publishing Co., p. $630,1975$.

DOYLE AE: Use of beta-adrenoceptor blocking drugs in hypertension. Drugs 8: 422, 1974.

DURÃO V, PRATA MM, PIRES GONÇALVES LM: Modification of antihypertensive effect of beta-adrenoceptor blocking agents by inhibition of endogenous prostaglandin synthesis. Lancet 2: 1005, 1977.

DURÃO V, JMGT RICO: Modification by indometracin of the blood pressure lowering effect of pindolol and propranolol in conscious rabbits. European / Pharmacol 43: 377, 1977.

DUSTING GJ, RAND MJ: An antihypertensive action of propranolol in DOCA/salt-treated rats. Clin Exptl Pharmacol Pbysiol 1: 87, 1974. 
ESLER M, JULIUS S, ZWEIGLER A, RANDALL O, HARBURG E, GARDINER H, DeQUAT. TRO V: Mild high-renin essential hypertension. Neurogenic human hypertension?. New Engl $J$ Med 296: 405, 1977.

FARMER JB, LEVY GP: A comparison of some cardiovascular properties of propanolol, M J 1999 and quinidine in relation to their effects in hypertensive animals. Br J Pharmacol 34: 116, 1968.

FURBERG C, MICHAELSON G: Effect of Aptin, a beta-adrenergic blocking agent in arterial hypertension. Acta Med Scand 186: 447, 1969.

GARVEY HL, RAM N: Comparative antihypertensive effects and tissue distribution of beta-adrenergic blocking drugs. J Pharmacol Exptl Therap 194: 220, 1975.

GIUDICELLI JF, SCHMITT H, BOISSIER JR: Studies on LB 46, a new potent beta-adrenergic blocking drug. I Pharmacol Exptl Therap 168: 116, 1969.

GOLDBLATT H, LYNCH J, HANZAL RF, SUMMERVILLE WW: Studies in experimental hypertension. 1. The production of persistent elevation in blood pressure by renal ischemia. I Exptl Med 59: 347, 1934.

GUEDON J: Hemodynamique de l'hypertension artérielle, Paris, eds. Merck Sharp and Dobme. p. 36,1974 .

IMBS JL, PARRENIN A, DESAULLES E, SCHWARTS J: Beta-blockers and renin secretion in anaesthetised dogs. Europ J Clin Invest 4: 324, 1974.

KELLEHER RT, MORSE WH, HERD JS: Effects of propranolol, phentolamine and methil atropine on cardiovascular function in the squirrel monkey during behavioural experiments. $J$ Pharmacol Exptl Therap 182: 204, 1972.

LANGER SZ: Presynaptic receptors and their role in the regulation of transmittor release. Brit J Pharmacol 60: 481, 1977.

LARAGH JH: Vasoconstrition - volume analysis for understanding and treating hypertension: the use of renin and aldosterone profiles. Amer I Med 55: 261, 1973.

LEE JB: Chemical and physiological properties of renal prostaglandins: the antihypertensive effects of medullin in essential human hypertension, in: Second Nobel Symposium, Prostaglandins, Stockholm, eds. S. Bertröm e B. Samuelson, 1967.

LEE JB: Prostaglandins and the renal antihypertentive and natriuretic endocrine function. Recent Progr Horm Res 30: 481, 1974.

LEE JB, COVINO BG, TAKMAN BH, SMITH ER: Renomedullary vasodepressor substance, medullin. Isolation, chemical characterization and physiological properties. Circ Res 17: 57, 1965.

LEE JB, HICKLER RB, SARAVIS CA, THORN GW: Sustained depressor effects of renomedullary extracts. Circulation 25: 747, 1962.

LEE JB, HICKLER RB, SARAVIS CA, THORN GW: Sustained depressor effect of renal medullary extract in the normotensive rat. Circ Res 13: 359. 1963.

LEE JB, MCGIFF JC, KANNEGIOSSER H: Prostaglandin $A_{1}$. Antihypertensive and renal effects. Am Intern Med 74: 703, 1971.

LEE JB, PATAK RV, MOOKERJEE BK: Renal prostaglandins and the regulation of blood pressure and sadium and water homeostasis. Amer J Med 60: 798, 1976.

LEE DR, SIMPSON FO: Effect of propanolol on blood pressure, heart rate and exchangeable sodium in genetically hypertensive and normotensive rats. Proc Univ Otago Med Sch 51: 51, 1973.

LEISHMAN AWD, THIRKETTLE JL, ALLEN BR, DIXON RA: Controlled trial of oxprenolol and practolol in hypertension. Brit Med J 4: 342, 1970.

LEWIS PJ, HEUSLER G: Reduction in sympathetic nervous activity as a mechanism for hypotensive effects of propranolol. Nature 256: 440, 1975.

MACKENNA DH, CORLISS RJ, SIALER S, ZARNSTORFF WC, CRUMPTON CW, ROWE GG: Effects of propranolol on systemic and coronary haemodynamics at rest and during stimulated exercise. Circulation Res 19: 520, 1966. 
MICHELAKIS AM, MCALLISTER RGJr: Renin secretion, adrenergic blockade and hypertension. Adv Exp Med Biol 17: 83, 1972.

PRATA MM, BARBAS JM, GUIMARÃES JA, DURÃO V: Estudo da aç̧ão hipotensora e avaliação do efeito sobre a actividade renina plasmática do pindolol em doentes com hipertensão essential, II Congresso Português de Cardiologia, Resumo das Comunicaçōes 60, 1977.

PRICHARD BNC: Hypotensive action of pronethalol. Brit Med J 1: 1227, 1964.

PRICHARD BNC, BOAKES AJ: The use of sotalol in the treatment of hypertension. In Advances in Beta-adrenergic Blocking Therapy. Sotatol. Smart Amsterdam Excerpta Med IV, 7, 1974.

PRICHARD BNC, GILLAM PMS: The use of propranolol in the treatment of hypertension. Br Med J 2: 725, 1964.

PRICHARD BNC, GILLAM PMS: Treatment of hypertension with propranolol. Br Med $J$ 1: 7, 1969.

SIMPSON FO: Beta-adrenergic receptor blocking drugs in hypertension. Drugs 7: 85, 1974.

STOKES GS, WEBER MA, THORNELL IR: Beta-Blockers and plasma renin activity in hypertension. $\mathrm{Br}$ Med J 1: 60, 1974.

TAKEDA K, SAKURAI H, IMAI S: Antihypertensive effects of beta-blockers in hypertensive rats. Jpn J Pharmacol 25: 82, 1975.

THORPE P: A controlled study of prindolol (Visken) in hypertension. Med J Aust 2: 306, 1972.

ULRYCH M: Changes of general haemodynamics during stressful mental arithmetic and non-stressing quiet conversation and modification of the later by beta-adrenergic blockade. Clin Sci 36: 453, 1969.

VAN ZWIETEN PA: The central action of anti-hypertensive drugs, mediated via central alpha-receptors. I Pharm Pbarmacol 25: 89, 1973.

WEBER MA, STOKES GS, GAIN JM: Comparison of the effects on renin release of beta-adrenergic antagonists with differing properties. I Clin Invest 54: 1413, 1974 a.

WEBER MA, THORNELL IR, STOKES GS: Effects of beta-adrenergic blocking agents on plasma renin activity in the conscious rabbit. I Pharmacol Exp Ther 188: 234, $1974 \mathrm{~b}$.

WOODS JW, PITTMAN A , PULLIAM CC, ERK Jr EE, WAIDER W, ALLEN CA: Renin profiling in hypertension and its use in treatment with propranolol and chlorthalidone. $N$ Engl J Med 294: 1137, 1976.

Pedido de Separatas: Virgílio Duräo

Instituto de Farmacologia

Faculdade de Medicina de Lisboa

Lisboa - Portugal 